\title{
Beta Streptococcus Group G
}

National Cancer Institute

\section{Source}

National Cancer Institute. Beta Streptococcus Group G. NCI Thesaurus. Code C86193.

A non-taxonomic grouping of beta hemolytic species within the Streptococcus genus

that are assigned to the G group. 